JOURNAL OF THE AMERICAN MATHEMATICAL SOCIETY

Volume 18, Number 1, Pages 217-237

S 0894-0347(04)00473-4

Article electronically published on November 18, 2004

\title{
ALTERNATING SIGNS OF QUIVER COEFFICIENTS
}

\author{
ANDERS SKOVSTED BUCH
}

\section{INTRODUCTION}

Let $X$ be a non-singular algebraic variety and $E_{0} \rightarrow E_{1} \rightarrow \cdots \rightarrow E_{n}$ a sequence of vector bundles and bundle maps over $X$. A set of rank conditions for this sequence is a collection $r=\left\{r_{i j}\right\}$ of non-negative integers, for $0 \leq i<j \leq n$. This data defines the quiver variety

$$
\Omega_{r}\left(E_{\bullet}\right)=\left\{x \in X \mid \operatorname{rank}\left(E_{i}(x) \rightarrow E_{j}(x)\right) \leq r_{i j} \forall i<j\right\},
$$

which comes with a natural structure of subscheme of $X$, given as the intersection of the zero sections of the maps $\bigwedge^{r_{i j}+1} E_{i} \rightarrow \bigwedge^{r_{i j}+1} E_{j}$. We demand that the rank conditions can occur as the ranks over a point in $X$. If we set $r_{i i}=\operatorname{rank}\left(E_{i}\right)$, then this is equivalent to the conditions that $r_{i j} \leq \min \left(r_{i, j-1}, r_{i+1, j}\right)$ for all $0 \leq$ $i<j \leq n$ and $r_{i j}+r_{i-1, j+1} \geq r_{i-1, j}+r_{i, j+1}$ for all $0<i \leq j<n$. In this case the expected codimension of the quiver variety $\Omega_{r}\left(E_{\bullet}\right)$ is the integer $d(r)=$ $\sum_{i<j}\left(r_{i, j-1}-r_{i j}\right)\left(r_{i+1, j}-r_{i j}\right)$.

In joint work with Fulton [10 we established a formula for the cohomology (or Chow) class of the quiver variety when this codimension is attained. This was generalized in [7] to the following formula for the structure sheaf of a quiver variety in the Grothendieck ring $K(X)$ of algebraic vector bundles on $X$ :

$$
\left[\mathcal{O}_{\Omega_{r}\left(E_{\bullet}\right)}\right]=\sum_{\mu} c_{\mu}(r) G_{\mu_{1}}\left(E_{1}-E_{0}\right) G_{\mu_{2}}\left(E_{2}-E_{1}\right) \cdots G_{\mu_{n}}\left(E_{n}-E_{n-1}\right) .
$$

Here the sum is over finitely many sequences $\mu=\left(\mu_{1}, \ldots, \mu_{n}\right)$ of partitions $\mu_{i}$ such that the sum $\sum\left|\mu_{i}\right|$ of the weights is greater than or equal to $d(r)$. The stable Grothendieck polynomials $G_{\mu_{i}}\left(E_{i}-E_{i-1}\right)$ are defined in Section 2. The quiver coefficients $c_{\mu}(r)$ appearing in this formula are integers which are uniquely determined by the condition that (1) is true for all varieties $X$ and bundle sequences $E_{\text {. }}$ together with the condition that $c_{\mu}(r)=c_{\mu}(r+m)$ holds for all $m \in \mathbb{N}$, where $r+m=\left\{r_{i j}+m\right\}$ are the rank conditions obtained by adding the integer $m$ to the original rank conditions.

A formula for quiver coefficients was also given in [10, 7]; in the case of $K$-theory, this is based on the algebra of stable Grothendieck polynomials constructed in [8]. Although the original formulas for quiver coefficients do not keep track of their signs, it was conjectured that the cohomological quiver coefficients (given by sequences $\mu$ such that $\left.\sum\left|\mu_{i}\right|=d(r)\right)$ are non-negative, while the $K$-theoretic quiver coefficients

Received by the editors January 5, 2004

2000 Mathematics Subject Classification. Primary 05E15; Secondary 14M15, 14M12, 19 E08.

Key words and phrases. Quiver variety, quiver coefficient, degeneracy locus, Grothendieck polynomial, Zelevinsky permutation, factor sequence.

(C)2004 American Mathematical Society Reverts to public domain 28 years from publication 
have signs that alternate with codimension, i.e. $(-1)^{\sum\left|\mu_{i}\right|-d(r)} c_{\mu}(r) \geq 0$. Special cases of these conjectures have been proved in [10, 5, 6, 7, 12, 13].

In their recent paper [24], Knutson, Miller, and Shimozono deliver a breakthrough within the theory of quiver formulas and prove at least two explicit combinatorial formulas for the cohomological quiver coefficients, which show that these coefficients are non-negative. One of the important ideas in their work is to reinterpret the lace diagrams of Abeasis and Del Fra 1 1 as sequences of partial permutations. This interpretation is explained by a Gröbner degeneration of a quiver variety in a matrix space into a union of products of matrix Schubert varieties. The component formula of 24 writes the cohomology class of a quiver variety as a sum, over all 'minimal' lace diagrams, of the products of the Schubert polynomials for the corresponding partial permutations. The proof that quiver coefficients are nonnegative is obtained by proving a stable version of this component formula, where the Schubert polynomials are replaced with Stanley symmetric functions. This is sufficient because Stanley symmetric functions are known to be Schur positive [15, 28].

Knutson, Miller, and Shimozono also prove a ratio formula, which writes the class of a quiver variety as a quotient of two Schubert polynomials. This formula follows from a careful analysis of the Zelevinsky map [34, 25] and is in fact established for both cohomology and $K$-theory. The component formulas are proved using a combination of the Gröbner degeneration and the ratio formula, as well as a combinatorial study of a double version of the ratio formula. In particular, it is proved that a limit of the double ratio formula agrees with the double quiver functions introduced in [10, 7] and named in [24]. The authors of [24] have informed us that they can generalize their methods to work in $K$-theory, although, according to their own description, this approach is rather complicated 1

In this paper we give simpler proofs of the above-mentioned formulas, using methods that work equally well in $K$-theory. In particular, we prove that the $K$-theoretic quiver coefficients have alternating signs, and we derive an explicit combinatorial formula for these coefficients. Starting from the ratio formula, we give combinatorial proofs of $K$-theoretic generalizations of the component formulas, where the Schubert polynomials and Stanley symmetric functions are replaced with ordinary and stable Grothendieck polynomials. These formulas are given in terms of sequences of partial permutations, which we call $K M S$-factorizations of the Zelevinsky permutation [34, 24]. To conclude that $K$-theoretic quiver coefficients have alternating signs and to derive the combinatorial formula for these coefficients, we use Lascoux's result that stable Grothendieck polynomials are linear combinations with alternating signs of stable Grothendieck polynomials for partitions [27]. To make our paper self-contained, we also give a short proof of the ratio formula.

The factor sequences conjecture of [10] states that cohomological quiver coefficients count the number of sequences of semistandard Young tableaux, which can be generated by a sequence of plactic factorizations and multiplications of chosen tableaux arranged in a tableaux diagram. A special case of this conjecture, corresponding to a particular choice of tableaux diagram, was proved in [24]. However, so far there has been no progress in generalizing this conjecture to $K$-theory. In this paper we close this gap by showing that KMS-factorizations can be defined by

\footnotetext{
${ }^{1}$ This work is now available in the paper [31], which proves a $K$-theoretic component formula and a double version of it, but only in the stable case and for large rank conditions.
} 
the same algorithm as defines factor sequences, except that the tableau diagram is replaced with a diagram of permutations and the plactic product of tableaux is replaced with multiplication of permutations in the degenerate Hecke algebra. It is also possible to formulate a new tableau-based version of the factor sequences conjecture. We briefly explain how our description of KMS-factorizations imply that cohomological quiver coefficients are counted by a new type of factor sequences of tableaux, which are generated using the Coxeter-Knuth product rather than the plactic product. In a paper in preparation with Kresch, Shimozono, Tamvakis, and Yong [11, the new tableau-based factor sequences have been generalized to work in $K$-theory.

We remark that it was already known that the cohomological component formulas can be derived combinatorially from the ratio formula, by using a simplification of Yong [33]. However, Yong's method still requires the analysis of the double ratio formula and its limits from [24. The approach presented here simplifies things further by working only with the single ratio formula, by applying Fomin and Kirillov's construction of Grothendieck polynomials based on solutions to Yang-Baxter equations [19, 18, and by observing that the stable component formula follows easily from the non-stable component formula.

Other simple proofs of the cohomological component formulas have also surfaced. For example, they can be deduced very easily from the Thom polynomial theory developed by Fehér and Rimányi [16] or deduced directly from the above-mentioned Gröbner degeneration with a symmetry argument. This is explained in 9]. While attempts to generalize these methods to $K$-theory have not been successful, they might hold more promise for quiver varieties of other types (see [16]).

Some of the results proved in [24] imply that the cohomological double ratio formula for large rank conditions satisfies nice properties, including multisupersymmetry and a double version of the component formula. In the last section of this paper, we establish these properties for the $K$-theoretic double ratio formula given by arbitrary rank conditions. In particular, we prove a conjecture from 24] stating that the double ratio formula satisfies a rank stability property. This conjecture is equivalent to the statement that the polynomials defined by the double ratio formula are specializations of the original quiver formulas [10, 7]. Even though the double ratio formula is not needed for the proof of alternating signs of quiver coefficients given in this paper, its multisupersymmetry property has some nice applications. For example, this property was used in 24] to prove the above-mentioned case of the original factor sequences conjecture. The multisupersymmetry property also implies that general quiver coefficients are special cases of the coefficients studied in [13. In fact, quiver coefficients can be realized as Schubert structure constants on flag varieties 3 , 30, 14].

This paper is organized as follows. In Section 2 we explain Fomin and Kirillov's construction of Grothendieck polynomials, which is a key ingredient in the combinatorial parts of this paper. Section 3 gives a new construction of the Zelevinsky permutation which is required for our proof that KMS-factorizations can be viewed as factor sequences. Section 4 contains the proof of the ratio formula. In Section 5 we prove a formula for double Grothendieck polynomials applied to certain rearrangements of the same set of variables, which in Section 6 is used to derive the non-stable component formula from the ratio formula. In Section 7 we establish the factor sequences definition of KMS-factorizations and discuss its consequences. 
As a corollary we obtain a rank stability property for KMS-factorizations, which in Section 8 is used to derive the stable component formula and deduce that quiver coefficients have alternating signs. Section 9 finally proves the above-mentioned properties of the double ratio formula.

We are very grateful to Richárd Rimányi for discussions at the Banach Center in Warsaw, which led to our observation that the stable component formula can be deduced from the non-stable formula and which triggered our search for other simplifications to 24. We also thank Fehér, Kresch, Sottile, Tamvakis, and Yong for inspiring collaboration on related papers, and Fulton, Miller, and Sottile for helpful comments about our paper. Finally, we thank Martin Guest, Anatol Kirillov, and the Research Institute for Mathematical Sciences in Kyoto for their hospitality while this paper was written.

\section{Grothendieck Polynomials}

The degenerate Hecke algebra $\mathcal{H}$ over a commutative ring $R$ is the free $R$-algebra generated by symbols $s_{1}, s_{2}, \ldots$, modulo the relations

$$
\begin{aligned}
s_{i} s_{j} & =s_{j} s_{i} \quad \text { if }|i-j| \geq 2, \\
s_{i} s_{i+1} s_{i} & =s_{i+1} s_{i} s_{i+1} \\
s_{i}^{2} & =-s_{i} .
\end{aligned}
$$

In this paper, $R$ will be a ring of Laurent polynomials. The algebra $\mathcal{H}$ is a free $R$-module with a basis of permutations corresponding to reduced expressions in the generators.

Given permutations $u_{1}, u_{2}, \ldots, u_{n}$, the product $u_{1} \cdot u_{2} \cdots u_{n}$ in $\mathcal{H}$ of these permutations is equal to plus or minus a single permutation $w$. We will call this permutation $w$ for the absolute Hecke product of the $u_{i}$. Notice that the descent positions of $w$, i.e. the indices $i$ for which $w(i)>w(i+1)$, include the descent positions of $u_{n}$, while the descent positions of $u_{1}^{-1}$ are also descent positions of $w^{-1}$. Notice also that if $u_{1} u_{2} \cdots u_{n}$ is a reduced product of permutations in the sense that $\ell\left(u_{1} u_{2} \cdots u_{n}\right)=\sum \ell\left(u_{i}\right)$, then the absolute Hecke product $w$ agrees with the usual product of permutations.

We will need Fomin and Kirillov's construction [19. 18] of the Grothendieck (Laurent) polynomials $\mathfrak{G}_{w}(a ; b)$ of Lascoux and Schützenberger [28, 26]. Consider a diagram $\mathfrak{D}$ of strings going from a top horizontal border to a left vertical border. Each string must be composed of line segments, each of which is labeled with a variable and has a direction between due south and due west. Furthermore, strings may only cross each other transversally, at inner points of the line segments. Of particular importance is the following diagram $\mathfrak{D}_{N}$, which contains only horizontal and vertical line segments.

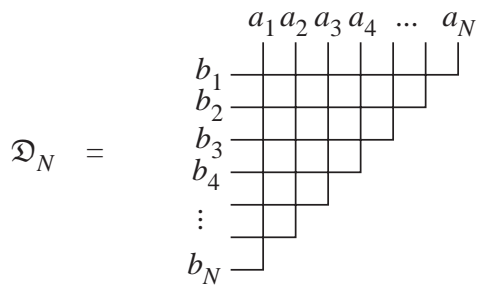


Let $C(\mathfrak{D})$ denote the set of crossing positions in the diagram $\mathfrak{D}$. For each $P \in$ $C(\mathfrak{D})$ we set $h(P)=1-\frac{y}{x}$ where $x$ is the label of the line through $P$ with the highest slope (within the range $[0,+\infty]$ ) and $y$ is the label of the line with the lowest slope. We also let $\nu(P)$ be one plus the number of strings in $\mathfrak{D}$ passing north-west of $P$. We then define the $F K$-product $\mathfrak{G}(\mathfrak{D})$ as the product in $\mathcal{H}$ of the factors $\left(1+h(P) s_{\nu(P)}\right)$ for all $P \in C(\mathfrak{D})$. These factors should be multiplied from south-west to north-east, in any order so that each crossing position $P$ comes before all other crossing positions in the quadrangle between the lines going due north and due east from $P$.

For the diagram $\mathfrak{D}_{N}$, we identify the crossing point of the horizontal line labeled $b_{p}$ and the vertical line labeled $a_{q}$, with the point $(p, q) \in \mathbb{N} \times \mathbb{N}$. We then have $\nu(p, q)=p+q-1$, and the FK-product of $\mathfrak{D}_{N}$ is given by

$$
\mathfrak{G}\left(\mathfrak{D}_{N}\right)=\prod_{q=1}^{N-1} \prod_{p=N-q}^{1}\left(1+\left(1-\frac{b_{p}}{a_{q}}\right) s_{p+q-1}\right) \in \mathcal{H} .
$$

We need the following theorem which is proved in 18 (modulo the change of variables $x_{i}=1-a_{i}^{-1}$ and $y_{i}=1-b_{i}$; see Thm. 2.3 and the remark on page 7 of loc. cit.)

Theorem 2.1 (Fomin and Kirillov). In $\mathcal{H}$ we have the identity

$$
\mathfrak{G}\left(\mathfrak{D}_{N}\right)=\sum_{w \in S_{N}} \mathfrak{G}_{w}(a ; b) \cdot w
$$

where $\mathfrak{G}_{w}(a ; b)$ is the double Grothendieck polynomial for $w$.

Suppose $D$ is a subset of the crossing positions $C(\mathfrak{D})$ of a diagram $\mathfrak{D}$. We let $w(D)$ be the absolute Hecke product of the simple reflections $s_{\nu(P)}$ for $P \in D$, in south-west to north-east order as above. We say that $D$ is an FK-graph for this permutation $w(D)$ and that $D$ is reduced if $|D|=\ell(w(D))$. We can picture an FK-graph $D$ by replacing the crossing positions of $\mathfrak{D}$ which belong to this graph with the symbol " $\frac{1}{\perp}$ ", while the remaining crossing positions are replaced with the symbol " $r$ ". Notice that if $D$ is reduced, then the string entering the resulting diagram at column $i$ at the top will exit at row $w(D)(i)$ at the left-hand side. Notice also that any FK-graph $D$ contains a reduced FK-graph $D^{\prime} \subset D$ such that $w\left(D^{\prime}\right)=w(D)$. In fact, $D^{\prime}$ can be found by simply skipping the points $P \in D$ for which $s_{\nu(P)}$ does not increase the length when the product $w(D)$ is formed.

When no diagram $\mathfrak{D}$ is explicitly mentioned, an FK-graph will always be relative to a diagram $\mathfrak{D}_{N}$, so it is a finite subset of $\mathbb{N} \times \mathbb{N}$. Such FK-graphs are called pipe dreams in [22], and a reduced pipe dream is the same as an $R C$-graph [2]. For example, the pipe dream

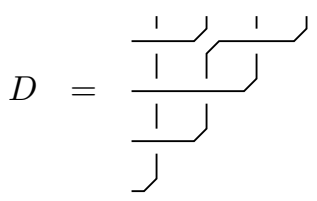

is an FK-graph for the permutation $w(D)=s_{3} s_{2} s_{1} s_{3}=4132$.

It follows from the definitions that the coefficient of a permutation $w$ in the FK-product of a diagram $\mathfrak{D}$ is equal to

$$
\sum_{D \subset C(\mathfrak{D}), w(D)=w}(-1)^{|D|-\ell(w)} \prod_{P \in D} h(P) .
$$


Theorem 2.1 therefore has the following corollary. See also [23] for an alternative proof and 2] for the case of Schubert polynomials.

Corollary 2.2. For any permutation $w$ we have

$$
\mathfrak{G}_{w}(a ; b)=\sum_{w(D)=w}(-1)^{|D|-\ell(w)} \prod_{(p, q) \in D}\left(1-\frac{b_{p}}{a_{q}}\right)
$$

where the sum is over all $F K$-graphs $D \subset \mathbb{N} \times \mathbb{N}$ for $w$.

Let us remark that Theorem 2.1 is more flexible than its corollary, as amply demonstrated in [19. The point is that many operations can be performed on a diagram $\mathfrak{D}$ without changing the corresponding FK-product. We will write $\mathfrak{D} \approx \mathfrak{D}^{\prime}$ if $\mathfrak{G}(\mathfrak{D})=\mathfrak{G}\left(\mathfrak{D}^{\prime}\right)$. The two key examples of this are

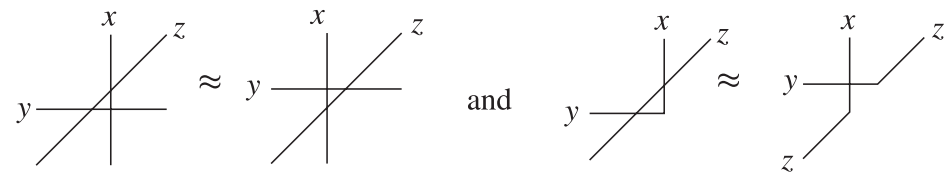

Notice that when $x=y$, the last diagram is also equivalent to a north to west hook labeled $x$ together with a disjoint north-east to south-west line labeled $z$. Although most diagrams in this paper contain only horizontal and vertical line segments, the availability of slanted lines often makes it more natural to manipulate these diagrams using the rules of (3).

Lascoux and Schützenberger's original definition of Grothendieck polynomials says that $\mathfrak{G}_{w_{0}}(a ; b)=\prod_{p+q \leq N}\left(1-b_{p} / a_{q}\right)$ where $w_{0} \in S_{N}$ is the longest permutation, and that $\left(a_{i}-a_{i+1}\right) \mathfrak{G}_{w}(a ; b)=a_{i} \mathfrak{G}_{w s_{i}}(a ; b)-a_{i+1} \mathfrak{G}_{w s_{i}}\left(a_{s_{i}} ; b\right)$ when $w(i)<w(i+1)$. Here we let $a_{v}$ denote the sequence of variables $a_{v(1)}, \ldots, a_{v(N)}$ for any permutation $v \in S_{N}$. It follows that if $i$ is not a descent position for $w$, then $\mathfrak{G}_{w}(a ; b)$ is symmetric in the variables $a_{i}$ and $a_{i+1}$. In particular, if $k$ is the last descent position for $w$, then the variables $a_{i}$ for $i>k$ do not occur in $\mathfrak{G}_{w}(a ; b)$. A similar relationship holds between the descent positions of $w^{-1}$ and the variables $b_{i}$. We need the identity

$$
\mathfrak{G}_{w}\left(a_{v(1)}, \ldots, a_{v(N)} ; a_{1}, \ldots, a_{N}\right)=\mathfrak{G}_{w_{0} w^{-1} w_{0}}\left(a_{N}, \ldots, a_{1} ; a_{v(N)}, \ldots, a_{v(1)}\right) .
$$

In fact, using that $\mathfrak{G}_{w}(a ; b)=\mathfrak{G}_{w^{-1}}\left(b_{1}^{-1}, \ldots, b_{N}^{-1} ; a_{1}^{-1}, \ldots, a_{N}^{-1}\right)$ and the above definition, equation (44) follows by descending induction on $\ell(w)$ from the calculation

$$
\begin{gathered}
\left(a_{v(i)}-a_{v(i+1)}\right) \mathfrak{G}_{w}\left(a_{v} ; a\right)=a_{v(i)} \mathfrak{G}_{w s_{i}}\left(a_{v} ; a\right)-a_{v(i+1)} \mathfrak{G}_{w s_{i}}\left(a_{v s_{i}} ; a\right) \\
=a_{v(i)} \mathfrak{G}_{w_{0} s_{i} w^{-1} w_{0}}\left(a_{w_{0}} ; a_{v w_{0}}\right)-a_{v(i+1)} \mathfrak{G}_{w_{0} s_{i} w^{-1} w_{0}}\left(a_{w_{0}} ; a_{v s_{i} w_{0}}\right) \\
=\left(a_{v(i)}-a_{v(i+1)}\right) \mathfrak{G}_{w_{0} w^{-1} w_{0}}\left(a_{w_{0}} ; a_{v w_{0}}\right) .
\end{gathered}
$$

The identity (4) also follows from the results in 29].

We also need the stable double Grothendieck polynomials $G_{w}(a ; b)$ of Fomin and Kirillov [18]. These polynomials are characterized by the property that

$$
G_{w}\left(a_{1}, \ldots, a_{q} ; b_{1}, \ldots, b_{p}\right)=\mathfrak{G}_{1^{m} \times w}\left(a_{1}, \ldots, a_{q}, 1, \ldots, 1 ; b_{1}, \ldots, b_{p}, 1, \ldots, 1\right)
$$

for all $m \geq \max (p, q)$. Here the permutation $1^{m} \times w$ is the identity on $\{1, \ldots, m\}$ while it maps $j$ to $w(j-m)+m$ for $j>m$. The polynomial $G_{w}(a ; b)$ is separately symmetric in its two sets of variables.

If $\lambda=\left(\lambda_{1} \geq \cdots \geq \lambda_{k}\right)$ is a partition, we set $G_{\lambda}(a ; b)=G_{w_{\lambda}}(a ; b)$ where $w_{\lambda}$ is the Grassmannian permutation for $\lambda$, defined by $w_{\lambda}(i)=i+\lambda_{k+1-i}$ for $1 \leq i \leq k$ 
and $w_{\lambda}(i)<w_{\lambda}(i+1)$ for $i \neq k$. It is proved in [8] that any stable Grothendieck polynomial $G_{w}(a ; b)$ can be written as an integral linear combination

$$
G_{w}(a ; b)=\sum_{\lambda} c_{w, \lambda} G_{\lambda}(a ; b)
$$

of stable Grothendieck polynomials for partitions. We will need a combinatorial formula of Lascoux for the coefficients $c_{w, \lambda}$ in this expansion, which shows that they have alternating signs.

Let $r$ be the last descent position of $w$, i.e. $r$ is maximal such that $w(r)>w(r+1)$. Set $w^{\prime}=w \tau_{r k}$ where $k>r$ is maximal such that $w(r)>w(k)$, and $\tau_{r k}$ is the transposition interchanging $r$ and $k$. We also set $I(w)=\left\{i<r \mid \ell\left(w^{\prime} \tau_{i r}\right)=\ell(w)\right\}$. Then define a relation $\triangleright$ on the set of all permutations as follows. If $I(w)=\emptyset$, then we write $w \triangleright v$ if and only if $v=1 \times w$. Otherwise we write $w \triangleright v$ if and only if there exist elements $i_{1}<\cdots<i_{p}$ of $I(w), p \geq 1$, such that $v=w^{\prime} \tau_{i_{1} r} \cdots \tau_{i_{p} r}$. The following result was proved in [27, Thm. 4].

Theorem 2.3 (Lascoux). The coefficient $c_{w, \lambda}$ of (15) is equal to $(-1)^{|\lambda|-\ell(w)}$ times the number of paths $w=w_{1} \triangleright w_{2} \triangleright \cdots \triangleright w_{m}$, such that $w_{m}=w_{\lambda}$ is a Grassmannian permutation for $\lambda$ and $w_{i}$ is not Grassmannian for $i<m$.

Given vector bundles $F=L_{1} \oplus \cdots \oplus L_{p}$ and $H=M_{1} \oplus \cdots \oplus M_{q}$ over $X$ which are direct sums of line bundles, we write $G_{\lambda}(H-F)=G_{\lambda}\left(M_{1}, \ldots, M_{q} ; L_{1}, \ldots, L_{p}\right) \in$ $K(X)$. By the symmetry of $G_{\lambda}$, this is a polynomial in the exterior powers of $F$ and $H^{\vee}$, so the notation $G_{\lambda}(H-F)$ also makes sense for bundles which are not sums of line bundles. This explains the notation used in the quiver formula (1).

\section{The Zelevinsky Permutation}

In this section we give a new construction of the Zelevinsky permutation [34, 24, which is needed for our proof of the $K$-theoretic analogue of the factor sequences conjecture. To be precise, we construct the conjugate of the Zelevinsky permutation defined in [24], which turns out to have a simpler relationship with the geometry of quiver varieties and KMS-factorizations. However, the Zelevinsky permutation itself is necessary to obtain nice combinatorial properties of the ratio formula.

Extend the set of rank conditions $r$ by setting $r_{i j}=e_{j}+e_{j+1}+\cdots+e_{i}$ for $j \leq i$, where $\left(e_{0}, \ldots, e_{n}\right)=\left(r_{00}, \ldots, r_{n n}\right)$ is the dimension vector corresponding to $r$. For $i<0$ or $j>n$ we set $r_{i j}=0$, and we set $N=r_{n 0}=e_{0}+\cdots+e_{n}$. For each $0 \leq i<n$ and $0<j \leq n$ we define a permutation $W_{i j} \in S_{r_{i+1, j-1}}$ by the expression

$$
W_{i j}(p)= \begin{cases}p+r_{i, j-1}-r_{i j} & \text { if } r_{i j}<p \leq r_{i+1, j} \\ p-r_{i+1, j}+r_{i j} & \text { if } r_{i+1, j}<p \leq r_{i+1, j}+r_{i, j-1}-r_{i j} \\ p & \text { otherwise. }\end{cases}
$$

When $i<j$, this is the Grassmannian permutation for the rectangular partition $R_{i j}$ from [10], with descent at position $r_{i+1, j}$. Now define the conjugate Zelevinsky permutation $z(r) \in S_{N}$ for the rank conditions $r$ to be the south-west to north-east product of the matrix of permutations

$$
\begin{array}{cccc}
W_{n-1,1} & W_{n-1,2} & \cdots & W_{n-1, n} \\
W_{n-2,1} & W_{n-2,2} & \cdots & W_{n-2, n} \\
\vdots & \vdots & \ddots & \vdots \\
W_{0,1} & W_{0,2} & \cdots & W_{0, n}
\end{array}
$$


that is, $z(r)=\prod_{j=1}^{n} \prod_{i=0}^{n-1} W_{i j}$. Notice that a 'south-west to north-east product' makes sense because $W_{i j}$ commutes with $W_{i^{\prime} j^{\prime}}$ whenever $i<i^{\prime}$ and $j^{\prime}<j$. Notice also that $W_{i j}$ is the conjugate Zelevinsky permutation for the set of rank conditions consisting of the integers $r_{i, j-1}, r_{i+1, j}$, and $r_{i j}$. Let $w_{0}^{(N)}$ denote the longest permutation in $S_{N}$. We will call the permutation $\widehat{z}(r)=w_{0}^{(N)} z(r)^{-1} w_{0}^{(N)}$ the Zelevinsky permutation for $r$, although the definition in 24 assigns this name to the inverse of $\widehat{z}(r)$. The action of $z(r)$ can be described explicitly as follows.

Lemma 3.1. Given any integer $1 \leq p \leq N$, there are unique integers $i$ and $j$ with $0 \leq i \leq j+1 \leq n+1$, such that $r_{n, j+1}+r_{i-1, j}-r_{i-1, j+1}<p \leq r_{n, j+1}+r_{i, j}-r_{i, j+1}$. We then have $z(r)(p)=p-r_{n, j+1}+r_{i, j+1}+r_{i-1,0}-r_{i-1, j}$.

Proof. To find $i$ and $j$, one first chooses $j$ such that $r_{n, j+1}<p \leq r_{n j}$, after which $i$ is uniquely determined. Now, when $z(r)$ is applied to $p$, the permutation $W_{k, j+1}$ subtracts $r_{k+1, j+1}-r_{k, j+1}$ from its argument for $k=n-1, n-2, \ldots, i$, after which $W_{i-1, k}$ adds $r_{i-1, k-1}-r_{i-1, k}$ to its argument for $k=j, j-1, \ldots, 1$. All other factors of $z(r)$ preserve their argument.

The relation of the Zelevinsky permutation with quiver varieties is based on the following lemma, which is equivalent to [24, Prop. 1.6].

Lemma 3.2. The conjugate Zelevinsky permutation $z(r)$ is the unique permutation in $S_{N}$ such that

(i) all descent positions of $z(r)$ are contained in the set $\left\{r_{n j} \mid 0<j \leq n\right\}$,

(ii) all descent positions of $z(r)^{-1}$ are contained in $\left\{r_{i 0} \mid 0 \leq i<n\right\}$, and

(iii) for all $0 \leq i, j \leq n$, there are $r_{i j}$ integers $p \leq r_{n j}$ for which $z(r)(p) \leq r_{i 0}$.

Proof. Part (i) follows directly from Lemma 3.1, and (ii) follows by observing that $z(r)^{-1}$ is the conjugate Zelevinsky permutation for the mirrored rank conditions $r^{\prime}$ given by $r_{i j}^{\prime}=r_{n-j, n-i}$. Notice that if $p, i, j$ are chosen as in Lemma 3.1, then $r_{i-1,0}<z(r)(p) \leq r_{i, 0}$. Therefore there are exactly $r_{i, j}-r_{i, j+1}-r_{i-1, j}+r_{i-1, j+1}$ integers $p$ such that $r_{n, j+1}<p \leq r_{n, j}$ and $r_{i-1,0}<z(r)(p) \leq r_{i, 0}$. This proves (iii) since $r_{i j}=\sum_{k=0}^{i} \sum_{l=j}^{n}\left(r_{k l}-r_{k, l+1}-r_{k-1, l}+r_{k-1, l+1}\right)$. The uniqueness statement is not needed in this paper, and its easy proof is left to the reader.

Lemma 3.2 implies that the length of $z(r)$ is given by

$$
\ell(z(r))=\sum_{0 \leq i<n, 0<j \leq n}\left(r_{i, j-1}-r_{i j}\right)\left(r_{i+1, j}-r_{i j}\right) .
$$

In fact, there are $\left(r_{i, j-1}-r_{i j}\right)\left(r_{i+1, j}-r_{i j}\right)$ pairs $(p, q)$ for which $p \leq r_{n j}<q \leq r_{n, j-1}$ and $z(r)(q) \leq r_{i 0}<z(r)(p) \leq r_{i+1,0}$. In particular, $z(r)$ is a reduced product of the permutations $W_{i j}$ of the matrix (6)).

We let $z(e)=z\left(r^{e}\right)$ be the conjugate Zelevinsky permutation for the maximal rank conditions $r^{e}$ given by $r_{i j}^{e}=\min \left\{e_{i}, e_{i+1}, \ldots, e_{j}\right\}$ for $i \leq j$. Similarly we write $\widehat{z}(e)=w_{0}^{(N)} z(e)^{-1} w_{0}^{(N)}$. The inverse of this permutation is called $v($ Hom $)$ in [24].

It follows from Lemma 3.1 that for all $i$ and $p>r_{n i}$ we have $z(r)(p) \leq r_{i 0}$. This implies that for $q \leq r_{n, i+1}$ we have $\widehat{z}(r)(q)=N+1-z\left(r^{\prime}\right)(N+1-q)>r_{i-1,0}$, so any FK-graph for $\widehat{z}(r)$ must contain the set $D_{e}=\bigcup_{i=1}^{n-1}\left[1, r_{i-1,0}\right] \times\left[1, r_{n, i+1}\right]$. Since $\left|D_{e}\right|=\sum_{j-i \geq 2} e_{i} e_{j}=\ell(\widehat{z}(e))$, it follows that $D_{e}$ is the unique FK-graph for $\widehat{z}(e)$. Corollary 2.2 therefore implies that the polynomial $\mathfrak{G}_{\widehat{z}(e)}(a ; b)=\prod_{(p, q) \in D_{e}}\left(1-\frac{b_{p}}{a_{q}}\right)$ divides $\mathfrak{G}_{\widehat{z}(r)}(a ; b)$ (cf. [24, $\left.\left.§ 5.1\right]\right)$. 


\section{The RAtio Formula}

In this section we give a coordinate-free proof of the ratio formula [24, Thm. 2.7]. The underlying geometry is similar to the original proof, but it becomes slightly simpler by working with the conjugate Zelevinsky permutation.

Suppose $F_{1} \subset F_{2} \subset \cdots \subset F_{N} \rightarrow H_{N} \rightarrow \cdots \rightarrow H_{2} \rightarrow H_{1}$ is a flag of vector bundles over $X$ with a morphism to a dual flag, such that $\operatorname{rank}\left(F_{i}\right)=\operatorname{rank}\left(H_{i}\right)=i$. For a permutation $w \in S_{N}$, Fulton [20] defines the degeneracy locus

$$
\Omega_{w}=\left\{x \in X \mid \operatorname{rank}\left(F_{q}(x) \rightarrow H_{p}(x)\right) \leq r_{w}(p, q) \forall p, q\right\}
$$

where $r_{w}(p, q)=\#\{k \leq p \mid w(k) \leq q\}$. This locus does not depend on the bundles $H_{p}$ for which $w(p)<w(p+1)$, or on the bundles $F_{q}$ such that $w^{-1}(q)<w^{-1}(q+1)$. The expected codimension of the locus is the length $\ell(w)$. We need the following formula for the Grothendieck class of $\Omega_{w}$, which generalizes Fulton's formula for its cohomology class [20. The $K$-theory formula was proved in [7, Thm. 2.1] as an application of [21, Thm. 3]. An equivalent statement was given in [22, Thm. A].

Theorem 4.1. If the codimension of $\Omega_{w}$ in $X$ equals $\ell(w)$, then

$$
\left[\mathcal{O}_{\Omega_{w}}\right]=\mathfrak{G}_{w}\left(L_{1}, \ldots, L_{N} ; M_{1}, \ldots, M_{N}\right) \in K(X)
$$

where $L_{i}=\operatorname{ker}\left(H_{i} \rightarrow H_{i-1}\right)$ and $M_{i}=F_{i} / F_{i-1}$.

It follows from Lemma 3.2 that the Grothendieck polynomial $\mathfrak{G}_{\widehat{z}(r)}(a ; b)$ is symmetric in each interval of variables $\left\{a_{p}: r_{n, i+1}<p \leq r_{n, i}\right\}$ and $\left\{b_{p}: r_{i-1,0}<\right.$ $\left.p \leq r_{i, 0}\right\}$. We therefore allow the vector bundle $E_{i}$ to be substituted for these intervals, and we interpret the result as if imaginative line bundle summands of $E_{i}$ had been inserted. We use the same notation in the quotient of polynomials $\mathfrak{G}_{\widehat{z}(r)}(a ; b) / \mathfrak{G}_{\widehat{z}(e)}(a ; b)$.

Theorem 4.2 (Knutson, Miller, Shimozono). If the codimension of $\Omega_{r}\left(E_{\bullet}\right)$ in $X$ is equal to $d(r)$, then the class of its structure sheaf in $K(X)$ is given by

$$
\left[\mathcal{O}_{\Omega_{r}\left(E_{\bullet}\right)}\right]=\frac{\mathfrak{G}_{\widehat{z}(r)}\left(E_{n}, \ldots, E_{0} ; E_{0}, \ldots, E_{n}\right)}{\mathfrak{G}_{\widehat{z}(e)}\left(E_{n}, \ldots, E_{0} ; E_{0}, \ldots, E_{n}\right)} .
$$

Proof. By replacing $X$ with $\bigoplus_{j-i \geq 2} \operatorname{Hom}\left(E_{i-1}, E_{i}\right)$, we may assume that there are general maps $\phi_{i j}: E_{i} \rightarrow E_{j}$ for all $i<j$, such that each map $\phi_{i-1, i}$ comes from the given bundle sequence $E_{\bullet}$. We may then construct the bundle sequence

$E_{0} \subset E_{0} \oplus E_{1} \subset \cdots \subset E_{0} \oplus \cdots \oplus E_{n} \stackrel{\phi}{\rightarrow} E_{0} \oplus \cdots \oplus E_{n} \rightarrow \ldots \rightarrow E_{n-1} \oplus E_{n} \rightarrow E_{n}$

where the middle map $\phi$ is composed of maps $E_{i} \rightarrow E_{j}$ which is $\phi_{i j}$ for $i<j$, the identity for $i=j$, and zero for $i>j$. All other maps are the obvious embeddings or projections. It follows from Lemma 3.2 that Fulton's locus $\Omega_{z(r)}$ for this sequence consists of the points in $X$ where each composed map $E_{0} \oplus \cdots \oplus E_{i} \rightarrow E_{j} \oplus \cdots \oplus E_{n}$ has rank at most $r_{i j}$.

We claim that $\Omega_{z(r)}$ is the intersection of the quiver variety $\Omega_{r}\left(E_{\bullet}\right)$ with the subset of $X$ where each map $\phi_{i j}$ is equal to the composition $\phi_{j-1, j} \phi_{j-2, j-1} \cdots \phi_{i, i+1}$. To see this, notice that the condition that the rank of the map $E_{0} \oplus \cdots \oplus E_{k} \rightarrow$ $E_{k} \oplus \cdots \oplus E_{n}$ is at most $e_{k}$ is equivalent to demanding that for all $i<k<j$ we have $\phi_{i j}=\phi_{k j} \phi_{i k}$. Given that this holds for all $k$, we obtain that for each $i<j$ the map $E_{0} \oplus \cdots \oplus E_{i} \rightarrow E_{j} \oplus \cdots \oplus E_{n}$ can be factored as a surjection followed by $\phi_{i j}$ followed by an injection, so the rank condition on this map is equivalent to 
$\operatorname{rank}\left(\phi_{i j}\right) \leq r_{i j}$. In particular, $\Omega_{z(e)}$ is the locus where $\phi_{i j}=\phi_{j-1, j} \cdots \phi_{i, i+1}$ for all $i<j$, and we have $\Omega_{z(r)}=\Omega_{r}\left(E_{\bullet}\right) \cap \Omega_{z(e)}$ as a scheme-theoretic intersection.

The above description also shows that the codimension of $\Omega_{z(r)}$ in $X$ is $\ell(z(r))=$ $d(r)+\ell(z(e))$, so by Theorem 4.1 and (4) we have $\left[\mathcal{O}_{\Omega_{z(r)}}\right]=\mathfrak{G}_{z(r)}\left(\breve{E}_{\bullet} ; E_{\bullet}\right)=$ $\mathfrak{G}_{\widehat{z}(r)}\left(\breve{E}_{\bullet} ; E_{\bullet}\right)$, where we write $\breve{E}_{\bullet}$ for the reversed sequence $E_{n}, E_{n-1}, \ldots, E_{0}$. Similarly we have $\left[\mathcal{O}_{\Omega_{z(e)}}\right]=\mathfrak{G}_{\widehat{z}(e)}\left(\breve{E}_{\bullet} ; E_{\bullet}\right)$. Since all of the degeneracy loci are CohenMacaulay [25] we obtain the identity

$$
\mathfrak{G}_{\widehat{z}(r)}\left(\breve{E}_{\bullet} ; E_{\bullet}\right)=\left[\mathcal{O}_{\Omega_{r}\left(E_{\bullet}\right)}\right] \cdot \mathfrak{G}_{\widehat{z}(e)}\left(\breve{E}_{\bullet} ; E_{\bullet}\right) .
$$

Comparing with (11), it follows that $\mathfrak{G}_{\widehat{z}(r)}\left(\breve{E}_{\bullet} ; E_{\bullet}\right)=\left(\sum_{\mu} c_{\mu}(r) \prod_{i} \mathfrak{G}_{\mu_{i}}\left(E_{i}-E_{i-1}\right)\right)$. $\mathfrak{G}_{\widehat{z}(e)}\left(\breve{E}_{\bullet} ; E_{\bullet}\right)$ on all varieties $X$, so this must hold as an identity of polynomials in the exterior powers of the bundles $E_{i}$. The theorem follows from this.

Remark. The above proof cites the main theorem of [7] for the existence of a universal polynomial that expresses the class of a quiver variety in the Grothendieck ring of an arbitrary variety $X$. When $X$ has an ample line bundle $L$, one can also deduce the theorem directly from (8) as follows. By twisting the sequence $E$. with a power of $L$, one may assume that all bundles $E_{i}$ are globally generated. In this case, one can construct a bundle $Y=\bigoplus_{i=1}^{n} \operatorname{Hom}\left(F_{i-1}, F_{i}\right)$ over a product of Grassmannians $\prod_{i=0}^{n} \operatorname{Gr}^{e_{i}}\left(\mathbb{C}^{k}\right)$ with tautological quotient bundles $F_{0}, \ldots, F_{n}$, such that the sequence $E_{\bullet}$ is the pullback of the universal sequence $F_{0} \rightarrow \cdots \rightarrow F_{n}$ on $Y$ along a map of varieties $f: X \rightarrow Y$. On $Y$ we know that $\mathfrak{G}_{\widehat{z}(r)}\left(\breve{F}_{\bullet} ; F_{\bullet}\right)=\left[\mathcal{O}_{\Omega_{r}\left(F_{\bullet}\right)}\right]$. $\mathfrak{G}_{\widehat{z}(e)}\left(\breve{F}_{\bullet} ; F_{\bullet}\right)$. By taking $k$ sufficiently large, we may therefore assume that the Grothendieck class of $\Omega_{r}\left(F_{\bullet}\right)$ agrees with $\mathfrak{G}_{\widehat{z}(r)}\left(\breve{F}_{\bullet} ; F_{\bullet}\right) / \mathfrak{G}_{\widehat{z}(e)}\left(\breve{F}_{\bullet} ; F_{\bullet}\right)$ in $K(Y) / I$, where $I \subset K(Y)$ is the ideal generated by the classes of subvarieties of codimension $\operatorname{dim}(X)$ or higher. The theorem follows from this because $f^{*}\left[\mathcal{O}_{\Omega_{r}\left(F_{\bullet}\right)}\right]=\left[\mathcal{O}_{\Omega_{r}\left(E_{\bullet}\right)}\right]$ and $f^{*}(I)=0$.

\section{Restricted FK-GRAPhs}

We will say that an FK-graph $D \subset \mathbb{N} \times \mathbb{N}$ is restricted w.r.t. the dimension vector $e=\left(e_{0}, \ldots, e_{n}\right)$ if for every point $(p, q) \in D$ and $0 \leq i \leq n$ we have $p \leq r_{i-1,0}$ or $q \leq r_{n, i+1}$.

For each $0 \leq i \leq n$ we let $a^{i}=\left(a_{1}^{i}, \ldots, a_{e_{i}}^{i}\right)$ be a set of $e_{i}$ variables. Set $a=\left(a^{0}, a^{1}, \ldots, a^{n}\right)=\left(a_{1}^{0}, \ldots, a_{e_{0}}^{0}, \ldots, a_{1}^{n}, \ldots, a_{e_{n}}^{n}\right)$ and $\breve{a}=\left(a^{n}, a^{n-1}, \ldots, a^{0}\right)=$ $\left(a_{1}^{n}, \ldots, a_{e_{n}}^{n}, \ldots, a_{1}^{0}, \ldots, a_{e_{0}}^{0}\right)$. We need the following variation of Corollary 2.2.

Corollary 5.1. For $w \in S_{N}$ we have

$$
\mathfrak{G}_{w}(\breve{a} ; a)=\sum_{D}(-1)^{|D|-\ell(w)} \prod_{(p, q) \in D}\left(1-\frac{a_{p}}{\breve{a}_{q}}\right)
$$

where the sum is over all FK-graphs $D$ for $w$, which are restricted w.r.t. e.

Proof. By Theorem 2.1, the Grothendieck polynomial $\mathfrak{G}_{w}(\breve{a} ; a)$ is the coefficient of $w$ in the FK-product of the diagram $\mathfrak{D}_{N}^{\prime}$ obtained from $\mathfrak{D}_{N}$ by replacing the top variables with $\breve{a}$ and the left side variables with $a$. For each crossing position $(p, q)$ in this diagram such that $a_{p}=\breve{a}_{q}$, the corresponding factor $\left(1+\left(1-\frac{a_{p}}{\breve{a}_{q}}\right) s_{p+q-1}\right)$ of $\mathfrak{G}\left(\mathfrak{D}_{N}^{\prime}\right)$ is equal to one. Therefore $\mathfrak{G}\left(\mathfrak{D}_{N}^{\prime}\right)$ is equal to the FK-product of the 
diagram obtained from $\mathfrak{D}_{N}^{\prime}$ by replacing these crossings with "ऽ" symbols. Now this diagram is the first of the following two equivalent diagrams:
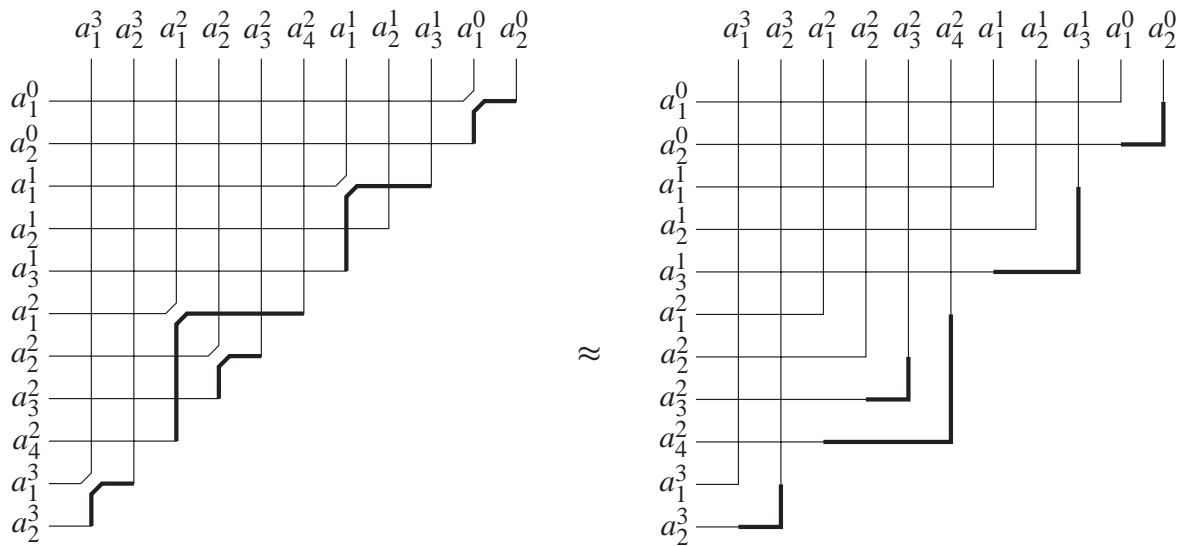

The equivalence follows by using the " $x=y$ " case of the second transformation of (3) to move the thickened line segments in the south-east direction. The corollary follows from (2) because $\mathfrak{G}_{w}(\breve{a} ; a)$ is the coefficient of $w$ in the FK-product of the second diagram.

\section{A $K$-Theoretic COMPONENT Formula}

For each $1 \leq j \leq n-1$ we set $\delta_{j}=W_{j j} W_{j+1, j} \cdots W_{n-1, j} \in S_{r_{n, j-1}}$. This is the Grassmannian permutation given by $\delta_{j}(p)=p+e_{j-1}$ for $e_{j}<p \leq r_{n j}$ and $\delta_{j}(p)=p-r_{n, j+1}$ for $r_{n j}<p \leq r_{n, j-1}$. We define a KMS-factorization for the rank conditions $r$ to be a sequence $\left(w_{1}, \ldots, w_{n}\right)$ of permutations with $w_{i} \in S_{e_{i-1}+e_{i}}$, such that the conjugate Zelevinsky permutation $z(r)$ is equal to the absolute Hecke product

$$
w_{1} \cdot \delta_{1} \cdot w_{2} \cdot \delta_{2} \cdots \delta_{n-1} \cdot w_{n}
$$

In the reduced case, these factorizations are equivalent to the minimal lace diagrams of Knutson, Miller, and Shimozono 24]2

A partial permutation contained in the rectangle $k \times l$ with $k$ rows and $l$ columns is a permutation $u \in S_{k+l}$, such that all descent positions of $u$ are less than or equal to $l$, while all descent positions of $u^{-1}$ are less than or equal to $k$. If this is true, then all FK-graphs for $u$ will be be contained in $[1, k] \times[1, l]$.

Lemma 6.1. If $\left(w_{1}, \ldots, w_{n}\right)$ is a KMS-factorization for the rank conditions $r$, then each permutation $w_{j}$ is a partial permutation contained in $e_{j-1} \times e_{j}$.

Proof. Since the absolute Hecke product $\alpha=w_{j+1} \cdot \delta_{j+1} \cdot w_{j+2} \cdots \delta_{n-1} \cdot w_{n}$ is a permutation in $S_{r_{n j}}$, we have $\delta_{j} \cdot \alpha(p)=\delta_{j}(p)=p-r_{n, j+1}$ for $r_{n j}<p \leq r_{n, j-1}$. If $w_{j}$ had a descent in the interval $\left[e_{j}+1, e_{j}+e_{j-1}-1\right]$, then the product (9) would obtain a descent in the interval $\left[r_{n j}+1, r_{n, j-1}-1\right]$, a contradiction. By using that $\left(w_{n}^{-1}, \ldots, w_{1}^{-1}\right)$ is a KMS-factorization for the mirrored rank conditions $r_{i j}^{\prime}=r_{n-j, n-i}$, we similarly deduce that $w_{j}^{-1}$ has no descent positions in the interval $\left[e_{j-1}+1, e_{j-1}+e_{j}-1\right]$.

\footnotetext{
${ }^{2}$ All permutations $w_{i}$ are inverted in the notation of [24].
} 
If $u$ is a partial permutation, and if the rectangle $k \times l$ is understood, we set $\widehat{u}=w_{0}^{(k+l)} u^{-1} w_{0}^{(k+l)}$, where $w_{0}^{(k+l)}$ is the longest element in $S_{k+l}$. Notice that the $180^{\circ}$ rotation of an FK-graph $D$ for $u$ will be an FK-graph for $\widehat{u}$. We will denote this rotated FK-graph by $\widehat{D}$, that is, $\widehat{D}=\{(k+1-p, l+1-q) \mid(p, q) \in D\}$.

Given a sequence $\left(P_{1}, \ldots, P_{n}\right)$ of FK-graphs such that each $P_{i}$ is contained in $\left[1, e_{i-1}\right] \times\left[1, e_{i}\right]$, we let $\widehat{\Phi}\left(P_{1}, \ldots, P_{n}\right)$ denote the FK-graph which is the union of the sets $\left\{\left(p+r_{i-2,0}, q+r_{n, i+1}\right) \mid(p, q) \in \widehat{P}_{i}\right\}$, for all $1 \leq i \leq n$, together with the unique FK-graph $D_{e}$ for the minimal Zelevinsky permutation $\widehat{z}(e)$.

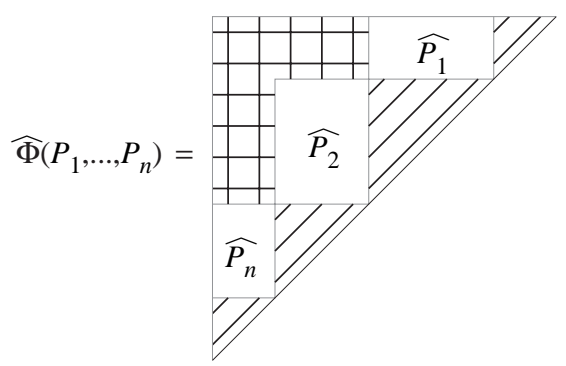

This construction was used in 24] (in the reverse direction) and in [33] (for certain special RC-graphs). Notice that the crossing positions to the left of $\widehat{P}_{i}$ in $\widehat{\Phi}\left(P_{1}, \ldots, P_{n}\right)$ form a reduced FK-graph for $w_{0}^{(N)} \delta_{i}^{-1} w_{0}^{(N)}$, and $D_{e}$ is the union of these positions.

Lemma 6.2. Let $r$ be a set of rank conditions. The map $\widehat{\Phi}$ gives a bijection of the set of sequences $\left(P_{1}, \ldots, P_{n}\right)$ of FK-graphs for which $\left(w\left(P_{1}\right), \ldots, w\left(P_{n}\right)\right)$ is a $K M S$-factorization for $r$, with the set of all restricted $F K$-graphs for $\widehat{z}(r)$.

Proof. The south-west to north-east absolute Hecke product of the simple reflections $s_{p+q-1}$ for $(p, q)$ in the block of $\widehat{P}_{i}$ is equal to $1^{N-e_{i}-e_{i-1}} \times w\left(\widehat{P}_{i}\right)=$ $w_{0}^{(N)} w\left(P_{i}\right)^{-1} w_{0}^{(N)}$. It follows from this that

$$
w_{0}^{(N)} w\left(\widehat{\Phi}\left(P_{1}, \ldots, P_{n}\right)\right)^{-1} w_{0}^{(N)}=w\left(P_{1}\right) \cdot \delta_{1} \cdot w\left(P_{2}\right) \cdot \delta_{2} \cdots \delta_{n-1} \cdot w\left(P_{n}\right) .
$$

The lemma therefore follows from Lemma 6.1 together with the definition (9) of KMS-factorizations.

We can now prove the following $K$-theoretic generalization of [24, Cor. 6.15].

Theorem 6.3. For any set of rank conditions $r$ we have

$\frac{\mathfrak{G}_{\widehat{z}(r)}(\breve{a} ; a)}{\mathfrak{G}_{\widehat{z}(e)}(\breve{a} ; a)}=\sum_{\left(w_{1}, \ldots, w_{n}\right)}(-1)^{\ell\left(w_{1} \cdots w_{n}\right)-d(r)} \mathfrak{G}_{w_{1}}\left(a^{1} ; a^{0}\right) \mathfrak{G}_{w_{2}}\left(a^{2} ; a^{1}\right) \cdots \mathfrak{G}_{w_{n}}\left(a^{n} ; a^{n-1}\right)$ where the sum is over all KMS-factorizations $\left(w_{1}, \ldots, w_{n}\right)$ for $r$.

Proof. Corollary 5.1 and Lemma 6.2 imply that $\mathfrak{G}_{\widehat{z}(r)}(\breve{a} ; a) / \mathfrak{G}_{\widehat{z}(e)}(\breve{a} ; a)$ is equal to

$$
\begin{aligned}
& \sum(-1)^{\ell\left(w_{1} \cdots w_{n}\right)-d(r)} \mathfrak{G}_{w_{1}}\left(a_{e_{1}}^{1}, \ldots, a_{1}^{1} ; a_{e_{0}}^{0}, \ldots, a_{1}^{0}\right) . \\
& \mathfrak{G}_{w_{2}}\left(a_{e_{2}}^{2}, \ldots, a_{1}^{2} ; a_{e_{1}}^{1}, \ldots, a_{1}^{1}\right) \cdots \mathfrak{G}_{w_{n}}\left(a_{e_{n}}^{n}, \ldots, a_{1}^{n} ; a_{e_{n-1}}^{n-1}, \ldots, a_{1}^{n-1}\right) .
\end{aligned}
$$

The theorem follows because $\mathfrak{G}_{\widehat{z}(r)}(\breve{a} ; a)$ is symmetric in each set of variables $a^{i}$. 
Remark. Let $\left(w_{1}, \ldots, w_{n}\right)$ be a sequence of partial permutations with $w_{i}$ contained in $e_{i-1} \times e_{i}$. Suppose $1 \leq j<n$ and $1 \leq k<e_{j}$ are given such that $w_{j}(k)<w_{j}(k+1)$ and $w_{j+1}^{-1}(k)<w_{j+1}^{-1}(k+1)$. If any of the sequences $\left(w_{1}, \ldots, w_{j} s_{k}, w_{j+1}, \ldots, w_{n}\right)$, $\left(w_{1}, \ldots, w_{j}, s_{k} w_{j+1}, \ldots, w_{n}\right)$, or $\left(w_{1}, \ldots, w_{j} s_{k}, s_{k} w_{j+1}, \ldots, w_{n}\right)$ is a KMS-factorization, then the definition shows that all three are KMS-factorizations. These transformations were first observed during an attempt to generalize the symmetry arguments of [9] to $K$-theory. In fact, in [9] it is proved that all KMS-factorizations of a given Zelevinsky permutation are connected by these transformations, which gives an easy way to find all of them.

\section{KMS-FACTORIZATIONS ARE FACTOR SEQUENCES}

We define the permutation diagram for the rank conditions $r$ to be the part of the matrix (6) which is on or below the antidiagonal.

$$
\begin{array}{cccc} 
& & & \\
& & & W_{n-1, n} \\
& & . & \vdots \\
W_{01} & W_{12} & \ldots & W_{1 n} \\
W_{02} & \ldots & W_{0 n}
\end{array}
$$

This diagram can also be obtained from the rectangle diagram of [10, §2.1] by replacing each rectangle $R_{i j}$ with the corresponding Grassmannian permutation $W_{i j}$ (and rotating the result 45 degrees counterclockwise.)

For $0 \leq k \leq n$ we let $r^{(k)}$ denote the rank conditions obtained from $r$ by dropping all integers $r_{i j}$ with $j-i<k$, that is, $r^{(k)}=\left\{r_{i j}^{(k)} \mid 0 \leq i \leq j \leq n-k\right\}$ where $r_{i j}^{(k)}=r_{i, j+k}$. The permutation diagram for these rank conditions is obtained from (10) by dropping the top $k$ diagonals of matrices $W_{i j}$ with $j-i \leq k$.

In this section we prove that all KMS-factorizations for $r$ can be obtained from the permutation diagram in the following way. First, if $n=1$, then the diagram has only one permutation $W_{0 n}$, and the only KMS-factorization is $\left(W_{0 n}\right)$. Otherwise any KMS-factorization can be obtained by first constructing a KMS-factorization $\left(\alpha_{1}, \ldots, \alpha_{n-1}\right)$ for $r^{(1)}$ and choosing arbitrary factorizations $\alpha_{i}=u_{i} \cdot v_{i}$ w.r.t. the absolute Hecke product. Then the sequence $\left(W_{01} \cdot u_{1}, v_{1} \cdot W_{12} \cdot u_{2}, \ldots, v_{n-1} \cdot W_{n-1, n}\right)$ is a KMS-factorization for the rank conditions $r$. The exact statement that we prove is the following theorem, which also includes a criterion for KMS-factorizations similar to the criterion for factor sequences proved in [5].

Theorem 7.1. (a) If $\left(w_{1}, \ldots, w_{n}\right)$ is a KMS-factorization for $r$, then each permutation $w_{i}$ has a reduced factorization $w_{i}=v_{i-1} \cdot W_{i-1, i} \cdot u_{i}$ with $v_{i-1} \in S_{e_{i-1}}$ and $u_{i} \in S_{e_{i}}$, such that $v_{0}=u_{n}=1$.

(b) Let $u_{1}, v_{1}, \ldots, u_{n-1}, v_{n-1}$ be permutations with $u_{i}, v_{i} \in S_{e_{i}}$. Then the sequence $\left(W_{01} \cdot u_{1}, v_{1} \cdot W_{12} \cdot u_{2}, \ldots, v_{n-1} \cdot W_{n-1, n}\right)$ is a KMS-factorization for $r$ if and only if $\left(u_{1} \cdot v_{1}, u_{2} \cdot v_{2}, \ldots, u_{n-1} \cdot v_{n-1}\right)$ is a KMS-factorization for $r^{(1)}$.

Based on this result, one can formulate a combinatorial formula for quiver coefficients in terms of a new type of factor sequences of tableaux. For cohomological quiver coefficients, this relies on Fomin and Greene's formula for Stanley coefficients [17] and uses a diagram of tableaux obtained from (10) by replacing $W_{i j}$ with the unique row and column decreasing tableau $T_{i j}$ of shape $R_{i j}$ (i.e. with $r_{i+1, j}-r_{i j}$ rows and $r_{i, j-1}-r_{i j}$ columns), such that the reading word of $T_{i j}$ is a reduced word 
for $W_{i j}$. Factor sequences are generated using the Coxeter-Knuth product, and it follows from Theorem 7.1, Corollary [8.2, and [17, Thm. 1.2] that a cohomological quiver coefficient $c_{\mu}(r)$ counts the number of factor sequences of shape $\mu$. A generalization of this formula to $K$-theoretic quiver coefficients has recently been proved with Kresch, Shimozono, Tamvakis, and Yong [11. This is based on a new formula for the coefficients $c_{w, \lambda}$ in the expansion of stable Grothendieck polynomials (5), which generalizes Fomin and Greene's result. We refer to [1] for details.

Let $0 \leq k<n$ and consider permutations $w_{1}, \ldots, w_{n-k}$ such that $w_{i} \in S_{r_{i, k+i-1}}$ for all $i$. We let $\Phi_{k}\left(w_{1}, \ldots, w_{n-k}\right) \in S_{N}$ denote the south-west to north-east product of the matrix obtained from (6) by replacing $W_{i-1, k+i}$ with $w_{i}$ for $1 \leq i \leq n-k$, and by replacing $W_{i j}$ with the identity for $j-i \geq k+2$. When $n=4$ and $k=1$ this matrix looks as follows:

$\begin{array}{cccc}W_{31} & W_{32} & W_{33} & W_{34} \\ W_{21} & W_{22} & W_{23} & w_{3} \\ W_{11} & W_{12} & w_{2} & 1 \\ W_{01} & w_{1} & 1 & 1\end{array}$

Notice that $\left(w_{1}, \ldots, w_{n}\right)$ is a KMS-factorization for $r$ if and only if $\Phi_{0}\left(w_{1}, \ldots, w_{n}\right)=$ $z(r)$. Furthermore, if $k>0$ and $u_{1}, v_{1}, \ldots, u_{n-k}, v_{n-k}$ are permutations such that $u_{i}, v_{i} \in S_{r_{i, k+i-1}}$, then $\Phi_{k}\left(u_{1} \cdot v_{1}, \ldots, u_{n-k} \cdot v_{n-k}\right)=\Phi_{k-1}\left(W_{0, k} \cdot u_{1}, v_{1} \cdot W_{1, k+1} \cdot u_{2}, \ldots, v_{n-k} \cdot W_{n-k, n}\right)$.

Lemma 7.2. If $\Phi_{k}\left(w_{1}, \ldots, w_{n-k}\right)=z(r)$, then each permutation $w_{i}$ has a reduced factorization $w_{i}=v \cdot W_{i-1, k+i} \cdot u$, where $v \in S_{r_{i-1, k+i-1}}$ and $u \in S_{r_{i, k+i}}$. Furthermore, $v$ is trivial when $i=1$ while $u$ is trivial when $i=n-k$.

Proof. Fix $i$ and set $j=k+i-1, a=r_{i-1, j}, b=r_{i, j+1}$, and $c=r_{i-1, j+1}$. For the first assertion it is enough to show that $w_{i} \in S_{a+b-c}$, that $w_{i}(p) \leq a$ for $b<p \leq a+b-c$, and that $w_{i}^{-1}(p) \leq b$ for $a<p \leq a+b-c$. In fact, $v w_{i} u$ will then satisfy the same properties for every $v \in S_{a}$ and $u \in S_{b}$. If we choose $v$ and $u$ such that $v^{-1} w_{i} u^{-1}$ has no descents in the interval $[1, b-1], u w_{i}^{-1} v$ has no descents in the interval $[1, a-1]$, and $\ell\left(v^{-1} w_{i} u^{-1}\right)=\ell\left(w_{i}\right)-\ell(v)-\ell(u)$, then we must have $v^{-1} w_{i} u^{-1}=W_{i-1, j+1}$, so we can use the factorization $w_{i}=v \cdot W_{i-1, j+1} \cdot u$.

Let $\sigma=W_{i-1,1} W_{i-1,2} \cdots W_{i-1, j}$ be the product of the permutations west of $w_{i}$ in the matrix (6), and let $\tau=\prod_{s=i}^{n-1} \prod_{t=1}^{j+1} W_{s t}$ be the product of the permutations weakly west and strictly north of $w_{i}$ in this matrix. It follows by induction on $j$ that all descent positions of $\sigma$ are greater than or equal to $r_{i j}$. A similar argument shows that the descent positions of $\tau^{-1}$ are greater than or equal to $r_{i j}$. Furthermore, the product $\sigma \tau$ is reduced, since it is part of the defining reduced factorization of the conjugate Zelevinsky permutation for rank conditions obtained by replacing $r_{i-1, j+1}$ with $\min \left(r_{i-1, j}, r_{i, j+1}\right)$. Since $w_{i} \in S_{r_{i j}}$, it follows that $\sigma w_{i} \tau$ is also a reduced product. We can therefore write $z(r)=\alpha \sigma w_{i} \tau \beta$ as a product of permutations, where $\alpha \in S_{r_{i-1,0}}$ and $\beta \in S_{r_{n, j+1}}$.

Notice that for $b<p \leq r_{i j}$ we have $\tau \beta\left(r_{n, j+1}-b+p\right)=\tau\left(r_{n, j+1}-b+p\right)=p$, and for $a<p \leq r_{i j}$ we have $\alpha \sigma(p)=\alpha\left(r_{i-1,0}-a+p\right)=r_{i-1,0}-a+p$. For $a+b-c<p \leq r_{i j}$ it follows from Lemma[3.1 that $z(r)\left(r_{n, j+1}-b+p\right)=r_{i-1,0}-a+p$, so we must have $w_{i}(p)=p$, that is, $w_{i} \in S_{a+b-c}$. The lemma also shows that $z(r)\left(r_{n, j+1}-b+p\right) \leq r_{i-1,0}$ for all $b<p \leq a+b-c$. This implies that $w_{i}(p) \leq a$, since otherwise we would have $\alpha \sigma w_{i} \tau \beta\left(r_{n, j+1}-b+p\right)=\alpha \sigma w_{i}(p)=r_{i-1,0}-a+w_{i}(p)>$ 
$r_{i-1,0}$. A symmetric argument shows that $w_{i}^{-1}(p) \leq b$ for $a<p \leq a+b-c$, which completes the proof of the first assertion.

When $i=n-k, \beta$ is trivial, so we have $\tau \beta(p)=p$ for all $p \leq b=r_{i n}$. If $u \in S_{b}$ was not the identity, then $z(r)$ would get a descent in the interval $\left[1, e_{n}-1\right]$, a contradiction. A similar argument shows that $v$ must be trivial when $i=1$.

Proof of Theorem 7.1. Part (a) is a special case of Lemma [7.2 so we prove part (b). By induction on $n$ we may assume that the theorem is true for all rank conditions $r^{(k)}$ with $k \geq 1$. We claim that $\left(w_{1}, \ldots, w_{n-k}\right)$ is a KMS-factorization for $r^{(k)}$ if and only if $\Phi_{k}\left(w_{1}, \ldots, w_{n-k}\right)=z(r)$. If either is true, then we can write $w_{i}=v_{i-1} \cdot W_{i-1, k+i} \cdot u_{i}$ for each $i$, where $u_{i}, v_{i} \in S_{r_{i, k+i}}$, and $v_{0}=u_{n-k}=1$. This proves the claim when $k=n-1$. For $1 \leq k \leq n-2$ we know by induction on $k$ that $\Phi_{k}\left(w_{1}, \ldots, w_{n-k}\right)=\Phi_{k+1}\left(u_{1} \cdot v_{1}, \ldots, u_{n-k-1} \cdot v_{n-k-1}\right)$ equals $z(r)$ if and only if $\left(u_{1} \cdot v_{1}, \ldots, u_{n-k-1} \cdot v_{n-k-1}\right)$ is a KMS-factorization for $r^{(k+1)}$, which by the theorem for $r^{(k)}$ is equivalent to $\left(w_{1}, \ldots, w_{n-k}\right)$ being a KMS-factorization for $r^{(k)}$. This proves the claim, and the theorem follows from the claim with $k=1$.

In the next section we need the following generalization of [24, Cor. 4.12].

Corollary 7.3. The KMS-factorizations for $r+m$ are precisely the sequences $\left(1^{m} \times w_{1}, \ldots, 1^{m} \times w_{n}\right)$ for which $\left(w_{1}, \ldots, w_{n}\right)$ is a KMS-factorization for $r$.

Proof. This is immediate from Theorem 7.1 because the permutation diagram for $r+m$ is obtained from (10) by replacing each permutation $W_{i j}$ with $1^{m} \times W_{i j}$.

\section{Alternating Signs of Quiver COefficients}

We can now prove the stable version of the component formula. We note that Theorem 9.3 of the next section can be substituted for the reference to geometry in its proof.

Theorem 8.1. For any set of rank conditions $r$ we have

$\frac{\mathfrak{G}_{\widehat{z}(r)}(\breve{a} ; a)}{\mathfrak{G}_{\widehat{z}(e)}(\breve{a} ; a)}=\sum_{\left(w_{1}, \ldots, w_{n}\right)}(-1)^{\ell\left(w_{1} \cdots w_{n}\right)-d(r)} G_{w_{1}}\left(a^{1} ; a^{0}\right) G_{w_{2}}\left(a^{2} ; a^{1}\right) \cdots G_{w_{n}}\left(a^{n} ; a^{n-1}\right)$ where the sum is over all KMS-factorizations for $r$.

Proof. It follows from (11) and Theorem 4.2 that $\mathfrak{G}_{\widehat{z}(r)}(\breve{a} ; a) / \mathfrak{G}_{\widehat{z}(e)}(\breve{a} ; a)$ is equal to $\sum_{\mu} c_{\mu}(r) G_{\mu_{1}}\left(a^{1} ; a^{0}\right) \cdots G_{\mu_{n}}\left(a^{n} ; a^{n-1}\right)$. Since $c_{\mu}(r)=c_{\mu}(r+m)$, we deduce that

$$
\frac{\mathfrak{G}_{\widehat{z}(r)}(\breve{a} ; a)}{\mathfrak{G}_{\widehat{z}(e)}(\breve{a} ; a)}=\frac{\mathfrak{G}_{\widehat{z}(r+m)}\left(a^{n}, 1^{m}, \ldots, a^{0}, 1^{m} ; a^{0}, 1^{m}, \ldots, a^{n}, 1^{m}\right)}{\mathfrak{G}_{\widehat{z}(e+m)}\left(a^{n}, 1^{m}, \ldots, a^{0}, 1^{m} ; a^{0}, 1^{m}, \ldots, a^{n}, 1^{m}\right)}
$$

for all integers $m \geq 0$. For $m \geq \max \left(e_{0}, \ldots, e_{n}\right)$ it follows from Theorem 6.3 and Corollary 7.3 that the right-hand side of this identity equals

$$
\begin{aligned}
& \sum(-1)^{\ell\left(w_{1} \ldots w_{n}\right)-d(r)} \mathfrak{G}_{1^{m} \times w_{1}}\left(a^{1}, 1^{m} ; a^{0}, 1^{m}\right) \cdots \mathfrak{G}_{1^{m} \times w_{n}}\left(a^{n}, 1^{m} ; a^{n-1}, 1^{m}\right) \\
& =\sum(-1)^{\ell\left(w_{1} \ldots w_{n}\right)-d(r)} G_{w_{1}}\left(a^{1} ; a^{0}\right) \cdots G_{w_{n}}\left(a^{n} ; a^{n-1}\right)
\end{aligned}
$$

as required.

It was conjectured in [7] that quiver coefficients have signs which alternate with codimension. This conjecture is a consequence of the following explicit formula for quiver coefficients, which follows from Theorem 8.1 and Lascoux's Theorem 2.3 
Corollary 8.2. The quiver coefficient $c_{\mu}(r)$ is given by

$$
c_{\mu}(r)=(-1)^{\sum\left|\mu_{i}\right|-d(r)} \sum_{\left(w_{1}, \ldots, w_{n}\right)}\left|c_{w_{1}, \mu_{1}} c_{w_{2}, \mu_{2}} \cdots c_{w_{n}, \mu_{n}}\right|
$$

where the sum is over all KMS-factorizations for the rank conditions $r$, and $c_{w_{i}, \mu_{i}}$ is defined by (5). Equivalently, $c_{\mu}(r)$ is equal to $(-1)^{\sum\left|\mu_{i}\right|-d(r)}$ times the number of sequences $\left(\gamma_{1}, \ldots, \gamma_{n}\right)$ of paths $\gamma_{i}=\left\{w_{i}=w_{i, 1} \triangleright \cdots \triangleright w_{i, m_{i}}\right\}$, such that $\left(w_{1}, \ldots, w_{n}\right)$ is a KMS-factorization for $r, w_{i, m_{i}}=w_{\mu_{i}}$ is a Grassmannian permutation for $\mu_{i}$, and $w_{i, j}$ is not Grassmannian for $1 \leq j<m_{i}$.

\section{Double Quiver POLYNOMIALS}

Let $r$ be a set of rank conditions. For each $0 \leq i \leq n$ we let $b^{i}=\left(b_{1}^{i}, \ldots, b_{e_{i}}^{i}\right)$ be a set of $e_{i}=r_{i i}$ variables, and we set $b=\left(b^{0}, b^{1}, \ldots, b^{n}\right)$. The $K$-theoretic analogue of the double ratio formula from [24] defines the polynomial

$$
K_{r}(a ; b)=\frac{\mathfrak{G}_{\widehat{z}(r)}(\breve{a} ; b)}{\mathfrak{G}_{\widehat{z}(e)}(\breve{a} ; b)},
$$

which is named a double quiver polynomial in 24]. In this section we prove some facts about such polynomials. In particular, we show that $K_{r}(a ; b)$ is a specialization of the quiver formula constructed in [7. When the rank conditions have the form $r+m$ for $m$ large, the cohomology versions of Theorems 9.19 .3 and 9.5 below follow from the results in 24 (see Cor. 6.13 and Thm. 6.20 in loc. cit.).

It follows from (i) and (ii) of Lemma 3.2 that $K_{r}(a ; b)$ is separately symmetric in each set of variables $a^{i}$ and $b^{i}$ (and that the variables $a^{0}$ and $b^{n}$ do not occur). In addition we have

Theorem 9.1. The polynomial $K_{r}(a ; b)$ is multisupersymmetric, that is, if one sets $a_{1}^{i+1}=b_{1}^{i}$ in $K_{r}(a ; b)$, then the result is independent of these variables.

Since $\mathfrak{G}_{\widehat{z}(e)}(\breve{a} ; b)=\prod_{(p, q) \in D_{e}}\left(1-\frac{b_{p}}{\breve{a}_{q}}\right)$, this theorem is an immediate consequence of the following proposition, applied to $\widehat{z}(r)$ with $k=r_{i-1,0}+1$ and $l=r_{n, i+2}+1$.

Proposition 9.2. Let $w \in S_{N}$ be a permutation and let $k, l \in \mathbb{N}$. Assume that $w(i)>k$ for $1 \leq i<l$ and that $w^{-1}(i)>l$ for $1 \leq i<k$. Then the polynomial

$$
\frac{\mathfrak{G}_{w}\left(a_{1}, \ldots, a_{l-1}, b_{k}, a_{l+1}, \ldots, a_{N} ; b_{1}, \ldots, b_{N}\right)}{\left(\prod_{i=1}^{k-1}\left(1-\frac{b_{i}}{b_{k}}\right)\right)\left(\prod_{i=1}^{l-1}\left(1-\frac{b_{k}}{a_{i}}\right)\right)}
$$

is independent of $b_{k}$.

Proof. By Theorem $2.1 \mathfrak{G}_{w}\left(a_{1}, \ldots, a_{l-1}, b_{k}, a_{l+1}, \ldots, a_{N} ; b_{1}, \ldots, b_{N}\right)$ is the coefficient of $w$ in the FK-product of the first of the following three equivalent diagrams:

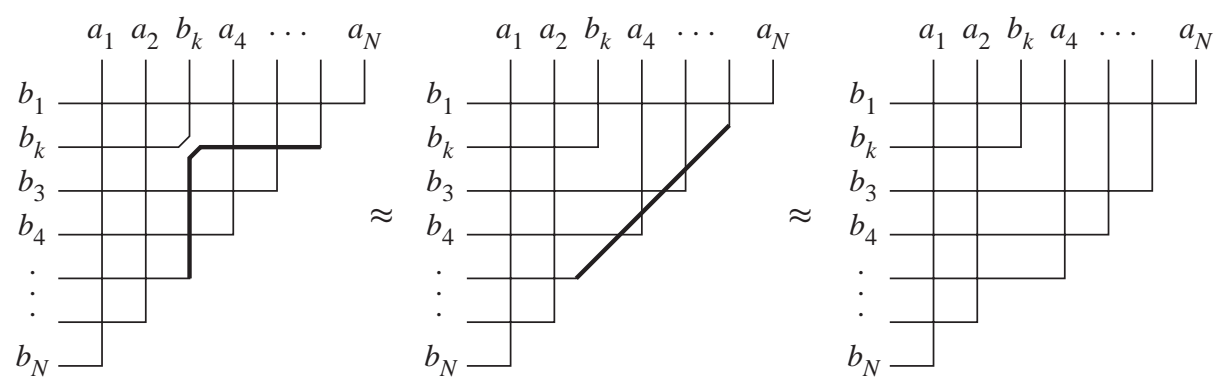


The first diagram is obtained from $\mathfrak{D}_{N}$ by replacing $a_{l}$ with $b_{k}$ and the crossing at position $(k, l)$ with " $\ulcorner$ ", and the others are the result of moving the thick line segments labeled $b_{k}$ in the south-east direction, using the rules of (3).

Notice that if $D$ is a subset of the crossing positions of the third diagram such that $w(D)=w$, then $D$ must contain all crossings involving the line segments labeled $b_{k}$. The proposition therefore follows from (2).

Our next theorem is a $K$-theoretic generalization of Conjecture 6.14 from [24]. It says that the double ratio formula satisfies rank stability.

Theorem 9.3. For any rank conditions $r$ and non-negative integer $m$ we have

$$
K_{r+m}\left(a^{0}, 1^{m}, \ldots, a^{n}, 1^{m} ; b^{0}, 1^{m}, \ldots, b^{n}, 1^{m}\right)=K_{r}\left(a^{0}, \ldots, a^{n} ; b^{0}, \ldots, b^{n}\right) .
$$

Proof. Using symmetry, it is enough to prove that

$$
\begin{aligned}
K_{r+1}\left(a^{0}, 1, c_{1}, a^{1}, \ldots, c_{n}, a^{n} ; c_{1}, b^{0}, \ldots, c_{n}, b^{n-1}, b^{n}, 1\right) \\
=K_{r}\left(a^{0}, \ldots, a^{n} ; b^{0}, \ldots, b^{n}\right) .
\end{aligned}
$$

The polynomial $\mathfrak{G}_{\widehat{z}(r+1)}=\mathfrak{G}_{\widehat{z}(r+1)}\left(c_{n}, a^{n}, \ldots, c_{1}, a^{1}, a^{0}, 1 ; c_{1}, b^{0}, \ldots, c_{n}, b^{n-1}, b^{n}, 1\right)$ is the coefficient of $\widehat{z}(r+1)$ in the FK-product of the following two equivalent diagrams (of size $N+n+1$ ):

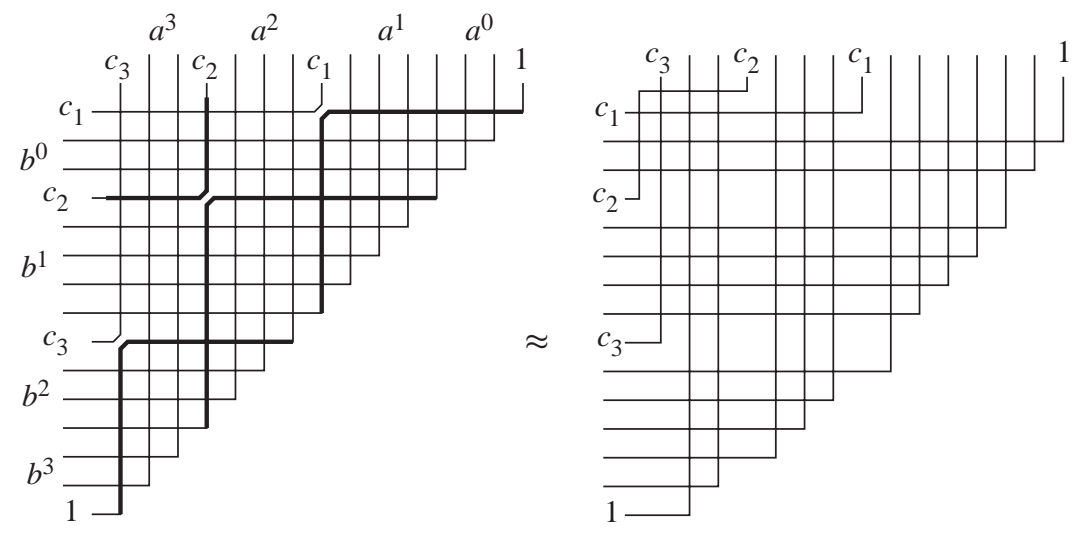

As indicated in the first diagram, the line segments extending furthest north and west are labeled with the variable sets $a^{i}$ and $b^{i}$, respectively. The equivalence is obtained by moving the thickened line segments toward the borders of the diagram. Since $\widehat{z}(r+1)$ fixes $N+n+1$, it follows that $\mathfrak{G}_{\widehat{z}(r+1)}$ is also the coefficient of $\widehat{z}(r+1)$ 
in the FK-product of the diagram:

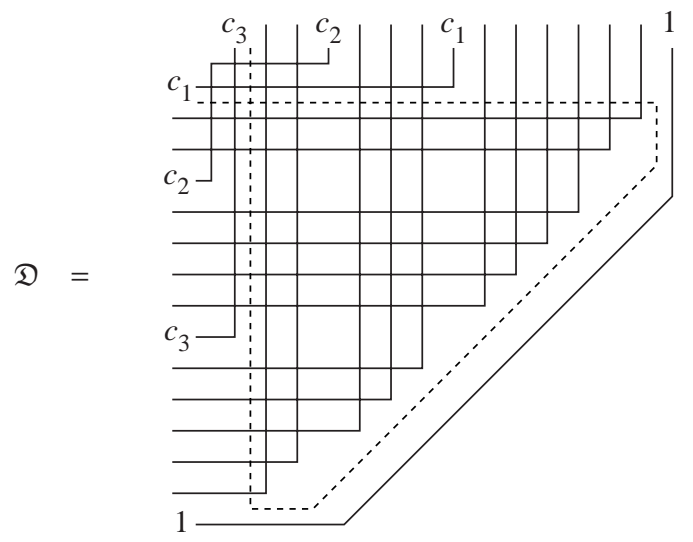

We claim that the FK-graphs for $\widehat{z}(r+1)$ w.r.t. this diagram are exactly those obtained by placing an FK-graph for $\widehat{z}(r)$ w.r.t. $\mathfrak{D}_{N}$ in the triangular region of $\mathfrak{D}$ and including all crossing positions $P$ outside this region. The theorem follows from this claim because it shows that $\mathfrak{G}_{\widehat{z}(r+1)}=\mathcal{Q} \cdot \mathfrak{G}_{\widehat{z}(r)}\left(a^{n}, a^{n-1}, \ldots, a^{0} ; b^{0}, b^{1}, \ldots, b^{n}\right)$, where $\mathcal{Q}$ is the product of the factors $h(P)$ for the crossing positions $P$ outside the triangular region, and (11) is immediate from this identity.

We will say that a permutation $w \in S_{N}$ satisfies the condition $\left(^{*}\right)$ for the set of rank conditions $r$, if for all $i$ and $p \leq r_{n, i+1}$ we have $w(p)>r_{i-1,0}$. As noted in Section $3, \widehat{z}(r)$ has this property. Notice that if $D \subset C(\mathfrak{D})$ is any FK-graph such that $w(D)$ satisfies $\left(^{*}\right)$ for $r+1$, then $D$ must contain all the crossing positions outside the triangular region. In particular, all FK-graphs for $\widehat{z}(r+1)$ must contain these crossing positions.

The crossing positions in the south-west region of $\mathfrak{D}$ represent the Grassmannian permutation $\alpha$ defined by $\alpha(i)=r_{i-2,0}+i$ for $1 \leq i \leq n$ and $\alpha(i)<\alpha(i+1)$ for $i \neq n$, while the permutation $\beta$ represented by the north-east crossings is given by $\beta^{-1}(i)=r_{n, n+2-i}+i$ for $1 \leq i \leq n$ and $\beta^{-1}(i)<\beta^{-1}(i+1)$ for $i \neq n$. If we let $w \in S_{N}$ be the permutation of an FK-graph placed in the triangular region of $\mathfrak{D}$, then the claim states that the absolute Hecke product $\alpha \cdot\left(w_{0}^{(n)} \times w\right) \cdot \beta$ equals $\widehat{z}(r+1)$ if and only if $w=\widehat{z}(r)$.

If $\alpha \cdot\left(w_{0}^{(n)} \times w\right) \cdot \beta$ satisfies $\left(^{*}\right)$ for $r+1$, then this product must be reduced. Otherwise one could skip a simple reflection factor of $\alpha$ or $\beta$, which could be exploited to construct an FK-graph for the product that missed the corresponding crossing outside the triangular region in $\mathfrak{D}$. Since a similar argument applies if the (usual) product of permutations $\alpha\left(w_{0}^{(n)} \times w\right) \beta$ satisfies $\left(^{*}\right)$ for $r+1$, it is enough to check that $\alpha\left(w_{0}^{(n)} \times w\right) \beta$ equals $\widehat{z}(r+1)$ if and only if $w=\widehat{z}(r)$.

Equivalently, we must check that the inverse of $\alpha\left(w_{0}^{(n)} \times \widehat{z}(r)\right) \beta$ maps $N+n+2-p$ to $N+n+2-z(r+1)(p)$ for every $2 \leq p \leq N+n+1$. We use Lemma 3.1 to do this. Set $r^{+}=r+1$. This means that $r_{i j}^{+}=r_{i j}+1$ for $i \leq j$ while $r_{i j}^{+}=r_{i j}+1+i-j$ when $i>j$. Now choose $0 \leq i \leq j+1 \leq n+1$ such that $r_{n, j+1}^{+}+r_{i-1, j}^{+}-r_{i-1, j+1}^{+}<p \leq r_{n, j+1}^{+}+r_{i, j}^{+}-r_{i, j+1}^{+}$. 
If $0 \leq j \leq n-1$ and $p=r_{n, j}^{+}$, then $i=j+1$ and $z\left(r^{+}\right)(p)=r_{j+1,0}^{+}$. Furthermore, $N+n+2-p=r_{j-1,0}+j+1$ is mapped to $j+1$ by $\alpha^{-1}$, which in turn is mapped to $n-j$ by $w_{0}^{(n)}$, and $\beta^{-1}(n-j)=r_{n, j+2}+n-j=N+n+2-r_{j+1,0}^{+}$, as required.

Otherwise we have $p \neq r_{n, j}^{+}$or $j=n$. Set $i^{\prime}=\max (i, 1)$ and $j^{\prime}=\min (j, n-1)$. Then $z\left(r^{+}\right)(p)=p+\delta+i^{\prime}+j^{\prime}-n$, where $\delta=-r_{n, j+1}+r_{i, j+1}+r_{i-1,0}-r_{i-1, j}$. Since $j^{\prime} \leq n-1$ is maximal such that $\alpha\left(j^{\prime}+1\right)<N+n+2-p$, we get $\alpha^{-1}(N+n+2-p)=$ $(N+n+2-p)+n-j^{\prime}-1$. One now checks that $r_{n, j+1}+r_{i-1, j}-r_{i-1, j+1}<$ $p-n+j^{\prime} \leq r_{n, j+1}+r_{i j}-r_{i, j+1}$. Using this, it follows that the inverse of $w_{0}^{(n)} \times \widehat{z}(r)$ $\operatorname{maps} \alpha^{-1}(N+n+2-p)=n+N+1-\left(p-n+j^{\prime}\right)$ to $n+N+1-z(r)\left(p-n+j^{\prime}\right)=$ $n+N+1-\left(p-n+j^{\prime}+\delta\right)$, and this number furthermore lies in the interval $\left[n+r_{n, i+1}+1, n+r_{n i}\right]$. Thus $\beta^{-1}$ subtracts $i^{\prime}-1$, so the inverse of $\alpha\left(w_{0}^{(n)} \times \widehat{z}(r)\right) \beta$ maps $N+n+2-p$ to $N+2 n+2-p-\delta-i^{\prime}-j^{\prime}$, as required.

We will finish this paper by proving that the double quiver polynomial $K_{r}(a ; b)$ is a specialization of the quiver formula of [7]. We need the following statement (about power series in the variables $x_{i}=1-a_{i}$ and $y_{i}=1-b_{i}$ ).

Lemma 9.4. Let $f(a ; b) \in \mathbb{Z}\left[a_{1}^{-1}, \ldots, a_{q}^{-1}, b_{1}, \ldots, b_{p}\right]$ be a Laurent polynomial that is separately symmetric in the variables $\left\{a_{i}\right\}$ and $\left\{b_{i}\right\}$. Assume furthermore that $f\left(c, a_{2}, \ldots, a_{q} ; c, b_{2}, \ldots, b_{p}\right)$ is independent of the variable $c$. Then $f$ is a (possibly infinite) linear combination of double stable Grothendieck polynomials $G_{\lambda}(a ; b)$.

Proof. If we set $b_{i}=1+y_{i}$ and $a_{i}=1+x_{i}$, that is, $\left(a_{i}\right)^{-1}=\sum_{k \geq 0}\left(-x_{i}\right)^{k}$, then the resulting power series $f\left(1+x_{i} ; 1+y_{i}\right)$ is supersymmetric, i.e. if one sets $x_{1}=y_{1}$, then $f\left(1+x_{i} ; 1+y_{i}\right)$ becomes independent of these variables. Since the lowest term of $G_{\lambda}\left(1+x_{i} ; 1+y_{i}\right)$ is the double Schur polynomial $s_{\lambda}(x ; y)$, it follows from 32 . Thm. 1] that there are coefficients $d_{\lambda} \in \mathbb{Z}$ such that the lowest term of

$$
f\left(1+x_{i} ; 1+y_{i}\right)-\sum d_{\lambda} G_{\lambda}\left(1+x_{i} ; 1+y_{i}\right)
$$

has higher degree than the lowest term of $f\left(1+x_{i} ; 1+y_{i}\right)$. The lemma follows from this because each $G_{\lambda}\left(1+x_{i} ; 1+y_{i}\right)$ is also supersymmetric [19].

We will prove in [4 that the linear combination in this lemma is always finite, but this fact is not needed for the proof of our last theorem.

Theorem 9.5. For any rank conditions $r$ we have

$$
K_{r}(a ; b)=\sum_{\mu} c_{\mu}(r) G_{\mu_{1}}\left(a^{1} ; b^{0}\right) G_{\mu_{2}}\left(a^{2} ; b^{1}\right) \cdots G_{\mu_{n}}\left(a^{n} ; b^{n-1}\right),
$$

where the sum is over all sequences $\mu=\left(\mu_{1}, \ldots, \mu_{n}\right)$ of partitions.

Proof. Theorem 9.1 and Lemma 9.4 imply that we can write

$$
K_{r+m}(a ; b)=\sum c_{\mu} G_{\mu_{1}}\left(a^{1} ; b^{0}\right) G_{\mu_{2}}\left(a^{2} ; b^{1}\right) \cdots G_{\mu_{n}}\left(a^{n} ; b^{n-1}\right)
$$

where the sum is over (possibly infinitely many) sequences of partitions $\mu$. Theorem 9.3 implies that the coefficients $c_{\mu}$ are independent of $m$. By setting $b^{i}=a^{i}$ for all $i$, it therefore follows from Theorem 4.2 and the definition (11) of quiver coefficients that $c_{\mu}=c_{\mu}(r)$ for all $\mu$. The result is obtained by setting $m=0$. 
By using Corollary [8.2, the above theorem can also be interpreted as a double component formula, that is,

$$
K_{r}(a ; b)=\sum_{\left(w_{1}, \ldots, w_{n}\right)}(-1)^{\ell\left(w_{1} \cdots w_{n}\right)-d(r)} G_{w_{1}}\left(a^{1} ; b^{0}\right) G_{w_{2}}\left(a^{2} ; b^{1}\right) \cdots G_{w_{n}}\left(a^{n} ; b^{n-1}\right),
$$

where the sum is over all KMS-factorizations for $r$.

Theorem 9.3 makes it possible to extend the quiver polynomial $K_{r}(a ; b)$ to infinite sets of variables $a^{i}$ and $b^{i}$, by taking the limit of $K_{r+m}(a ; b)$ as $m$ tends to infinity. Such limits are called double quiver functions in 24. In the $K$-theory case it is preferable to change to the variables $x_{j}^{i}=1-\left(a_{j}^{i}\right)^{-1}$ and $y_{j}^{i}=1-b_{j}^{i}$ in order to obtain a nice formal power series. This recovers the quiver formula $P_{r}$ constructed in [7, §4]. In fact, Theorem 9.5 shows that the function $\lim _{m \rightarrow \infty} K_{r+m}(a ; b)$ is obtained from $P_{r}$ by setting $1^{\otimes i-1} \otimes x_{j} \otimes 1^{\otimes n-i}=1-\left(a_{j}^{i}\right)^{-1}$ and $1^{\otimes i-1} \otimes y_{j} \otimes 1^{\otimes n-i}=$ $1-b_{j}^{i-1}$. In particular, the cohomological double quiver function used in [24] is equal to the lowest term of $P_{r}$. Equivalently, this cohomological quiver function is a specialization of the original quiver formula from [10 (see also the construction of this formula given in [5, §2]).

By setting the variables $b_{j}^{i}$ equal to 1 in Theorem 9.5, one can deduce that general quiver coefficients are special cases of the coefficients studied in [13. More details about this will be given in [14, together with a proof that quiver coefficients are special cases of Schubert structure constants (see also [3, 30]).

\section{REFERENCES}

[1] S. Abeasis and A. Del Fra, Degenerations for the representations of an equioriented quiver of type $A_{m}$, Boll. Un. Mat. Ital. Suppl. (1980), no. 2, 157-171. MR0675498 (84e:16019)

[2] N. Bergeron and S. Billey, RC-graphs and Schubert polynomials, Experiment. Math. 2 (1993), no. 4, 257-269. MF 1281474 (95g:05107)

[3] N. Bergeron and F. Sottile, Schubert polynomials, the Bruhat order, and the geometry of flag manifolds, Duke Math. J. 95 (1998), no. 2, 373-423. MR1652021 (2000d:05127)

[4] A. S. Buch, Supersymmetry of Grothendieck polynomial, in preparation.

[5] - On a conjectured formula for quiver varieties, J. Algebraic Combin. 13 (2001), no. 2, 151-172. MR.1826950 (2002g:14074)

[6] _ Stanley symmetric functions and quiver varieties, J. Algebra 235 (2001), no. 1, 243-260. MR 1807664 (2001m:05257)

[7] _ Grothendieck classes of quiver varieties, Duke Math. J. 115 (2002), no. 1, 75-103. MR $1932326(2003 \mathrm{~m}: 14018)$

[8] _ A Littlewood-Richardson rule for the K-theory of Grassmannians, Acta Math. 189 (2002), no. 1, 37-78. MR1946917 (2003j:14062)

[9] A. S. Buch, L. M. Fehér, and R. Rimányi, Positivity of quiver coefficients through Thom polynomials, to appear in Adv. Math., 2003.

[10] A. S. Buch and W. Fulton, Chern class formulas for quiver varieties, Invent. Math. 135 (1999), no. 3, 665-687. MR.1669280 (2000f:14087)

[11] A. S. Buch, A. Kresch, M. Shimozono, H. Tamvakis, and A. Yong, Stable Grothendieck polynomials and $K$-theoretic factor sequences, in preparation.

[12] A. S. Buch, A. Kresch, H. Tamvakis, and A. Yong, Schubert polynomials and quiver formulas, Duke Math. J. 122 (2004), no. 1, 125-143. MR2046809

[13] _ Grothendieck polynomials and quiver formulas, To appear in Amer. J. Math., 2003.

[14] A. S. Buch, F. Sottile, and A. Yong, Quiver coefficients are Schubert structure constants, preprint, 2003.

[15] P. Edelman and C. Greene, Balanced tableaux, Adv. in Math. 63 (1987), no. 1, 42-99. MR 0871081 (88b:05012)

[16] L. Fehér and R. Rimányi, Classes of degeneracy loci for quivers: the Thom polynomial point of view, Duke Math. J. 114 (2002), 193-213. MR.1920187|(2003j:14005) 
[17] S. Fomin and C. Greene, Noncommutative Schur functions and their applications, Discrete Math. 193 (1998), no. 1-3, 179-200, Selected papers in honor of Adriano Garsia (Taormina, 1994). MR.1661368(2000c:05149)

[18] S. Fomin and A. N. Kirillov, Grothendieck polynomials and the Yang-Baxter equation, Proc. Formal Power Series and Alg. Comb. (1994), 183-190.

[19] - The Yang-Baxter equation, symmetric functions, and Schubert polynomials, Discrete Math. 153 (1996), 123-143. MR.1394950 (98b:05101)

[20] W. Fulton, Flags, Schubert polynomials, degeneracy loci, and determinantal formulas, Duke Math. J. 65 (1992), no. 3, 381-420. MF 1154177 (93e:14007)

[21] W. Fulton and A. Lascoux, A Pieri formula in the Grothendieck ring of a flag bundle, Duke Math. J. 76 (1994), no. 3, 711-729. MF.1309327 (96j:14036)

[22] A. Knutson and E. Miller, Gröbner geometry of Schubert polynomials, to appear in Ann. of Math. (2), 2003.

[23] _ Subword complexes in Coxeter groups, Adv. Math. 184 (2004), 161-176. MR2047852

[24] A. Knutson, E. Miller, and M. Shimozono, Four positive formulas for type A quiver polynomials, preprint, 2003.

[25] V. Lakshmibai and P. Magyar, Degeneracy schemes, quiver schemes, and Schubert varieties, Internat. Math. Res. Notices 1998, no. 12, 627-640. MR/1635873 (99g:14065)

[26] A. Lascoux, Anneau de Grothendieck de la variété de drapeaux, The Grothendieck Festschrift, Vol. III, Birkhäuser Boston, Boston, MA, 1990, pp. 1-34. MR1106909 (92j:14064)

[27] - Transition on Grothendieck polynomials, Physics and combinatorics, 2000 (Nagoya), World Sci. Publishing, River Edge, NJ, 2001, pp. 164-179. MR1872255 (2002k:14082)

[28] A. Lascoux and M.-P. Schützenberger, Structure de Hopf de l'anneau de cohomologie et de l'anneau de Grothendieck d'une variété de drapeaux, C. R. Acad. Sci. Paris Sér. I Math. 295 (1982), no. 11, 629-633. MR0686357 (84b:14030)

[29] _ Décompositions dans l'algèbre des différences divisées, Discrete Math. 99 (1992), no. 1-3, 165-179. MR.1158787 (93c:20030)

[30] C. Lenart, S. Robinson, and F. Sottile, Grothendieck polynomials via permutation patterns and chains in the Bruhat order, preprint, 2003.

[31] E. Miller, Alternating formulae for $K$-theoretic quiver polynomials, to appear in Duke Math. J., 2003.

[32] J. R. Stembridge, A characterization of supersymmetric polynomials, J. Algebra 95 (1985), no. 2, 439-444. MF 0801279 (87a:11022)

[33] A. Yong, On combinatorics of quiver component formulas, to appear in J. Algebraic Combin., 2003.

[34] A. V. Zelevinskiı̌, Two remarks on graded nilpotent classes, Uspekhi Mat. Nauk 40 (1985), no. 1(241), 199-200. MR0783619(86e:14027)

Matematisk Institut, Aarhus Universitet, Ny Munkegade, 8000 Århus C, Denmark E-mail address: abuch@imf.au.dk 